\title{
Zwischen Enthemmung und Autoritarismus: Deutschland in der Mitte
}

\author{
Ein Gespräch mit Oliver Decker
}

Zur Person: PD Dr. phil. Oliver Decker ist Sozialpsychologe und Leiter des Forschungsbereichs Gesellschaftlicher und medizinischer Wandel an der Selbstständigen Abteilung für Medizinische Psychologie und Medizinische Soziologie der Universität Leipzig. Seit 2013 ist er zudem als Direktor des Kompetenzzentrums für Rechtsextremismus- und Demokratieforschung sowie seit 2018 als Co-Sprecher des Graduiertenprogramms «Rechtspopulismus» der Universität Leipzig engagiert. Im Sommersemester 2019 weilte er als Gastprofessor am Institut für Soziologie der Universität Wien. Des Weiteren ist er u.a. Gründer und Geschäftsführender Herausgeber der Zeitschrift «Psychoanalyse - Texte zur Sozialforschung" und Mit-Herausgeber der Zeitschrift "Psychosozial» wie im internationalen Board des "Journal for Psychosocial Studies». 2018 erschien das von ihm herausgebende, zweibändige Lehrbuch «Sozialpsychologie und Sozialtheorie» im Springer-Verlag.

Für die Journalgruppe entwarfen Marie-Luise Hermann, Laura Wolf und Norbert Wolff die Fragen. Das Interview führte Laura Wolf im Dezember 2018, Ergänzungen folgten im März 2019.

Schlüsselwörter:Enthemmung, Autoritarismus, Deutschland, psychoanalytische Sozialforschung

Laura Wolf: Von 2002 bis 2016 sind im Zweijahresrhythmus die bekannten «Mitte»-Studien - seit 2018 unter dem Titel «Leipziger Autoritarismus-Studie» zu rechtsextremen Einstellungen in Deutschland von Dir und Elmar Brähler leitend durchgeführt worden. In diesen unternimmt Eure Arbeitsgruppe repräsentative Erhebungen wie auch Gruppendiskussionen, die zudem die Entstehung der Einstellungen untersuchen. Das Besondere ist, dass Ihr Euch die sogenannte Mitte der Gesellschaft anschaut. Wie definiert Ihr «die Mitte»? 
Oliver Decker: Das ist ein sehr guter Einstieg, denn wir wurden und werden für unsere Verwendung des Mitte-Begriffs häufig angegangen. Vermutlich weil wir an einer hoch aufgeladenen, ideologischen Figur an zentraler Stelle des bundesrepublikanischen Selbstverständnisses gerührt haben. Um die Funktion des Mitte-Begriffs zu verstehen, kann man noch weiter in der Geschichte des europäischen Denkens zurückgehen, denn diese Mitte-Figur ist eigentlich schon sehr alt. So finden wir sie in ihrer Bedeutungsdimension bereits bei Aristoteles in der Nikomachischen Ethik beschrieben. Nach heutigen Begriffen hat Aristoteles die Mitte jeweils auf einer horizontalen und einer vertikalen Ebene beschrieben. Erstere adressiert die Ausprägung von Persönlichkeitseigenschaften, die bei Menschen mehr oder weniger stark vorliegen. Aristoteles' Forderung war die nach Mässigung, nach einem sittlichen Handeln das sich weder durch zu starke noch zu schwache Impulse auszeichnet. Man solle weder Feigling sein, noch sich zu stark in Kämpfe verwickeln lassen. Mit dieser moralischen Setzung, die einen deutlichen Appellcharakter aufweist, solle man für sein Begehren und sein Verhalten stets das richtige Mass finden. In der vertikalen Ebene beschreibt Aristoteles, dass eine Polis am besten aufgestellt sei, wenn die Mitte stark sei. Er meinte auf dieser Ebene die ökonomische Mitte. So soll weder der Plebs regieren, noch die übermässig Besitzenden. Beide gesellschaftlichen Gruppen wären nicht in der Lage, weit voraus zu schauen und hätten nicht das Gemeinwohl, sondern nur ihr Eigenwohl im Blick. Diejenigen, hingegen, die ihre ökonomische Position im Mittelmass hätten, seien besonders geeignet, die Polis zu regieren und deswegen würden Städte mit einer starken Mitte, so Aristoteles' Idee, auch am besten aufgestellt sein. Seine Vorstellung ist damit aber antidemokratisch und hat zum Anliegen, einen zu starken Einbezug zu vieler Leute zu diskreditieren. Dennoch war damit sowohl der Anrufungscharakter nach Mass und Mitte als Orientierung für eine sittliche Lebensführung, als auch die Idee einer stabilisierenden gesellschaftlichen Mitte in der Welt. Mit der Moderne kam dieser Bedeutungsgehalt auch räumlich zum Ausdruck: durch die Sitzordnung in der Nationalversammlung nach der französischen Revolution. Bekanntlich sass die Fraktion, die gerne die Revolution weitergetrieben hätte, vom Präsidium aus gesehen links, die restaurativen Kräfte sassen rechts und die Gemässigten in der Mitte. Auf diese Weise wurde der Begriff in den politischen Raum eingeführt und aus diesem kennen wir ihn hauptsächlich. Die "politische Mitte» ist in der Bundesrepublik der bekannteste und vertrauteste Begriff. Von diesem lassen sich, wie Aristoteles sich das bereits dachte, die extremen Ränder abgrenzen. Eine gemäßigte Mitte müsse stark sein, damit die gesellschaftliche Ordnung stabil und in unserem Falle, die Demokratie stark sei. 
Doch als wir unsere Untersuchungen begonnen haben, haben wir festgestellt, dass für die Bedrohung der Demokratie auf der Einstellungsebene von einer Begrenzung auf irgendwelche Ränder nicht die Rede sein kann, sondern dass Ressentiments in allen gesellschaftlichen Schichten und in einem grossen Teil der Bevölkerung zu finden sind. Die Anerkennung des Anderen ist aber die Voraussetzung einer liberalen Demokratie. Und diese antidemokratischen Einstellungen lassen sich nicht auf den rechtsextremen Rand begrenzen, sie sind in verschiedenen gesellschaftlichen Gruppen vorhanden. Durch diesen Befund und der damit einhergehenden Kritik am Extremismusbegriff kamen wir zu der Ansicht, dass es keinesfalls so ist, dass «die (gesellschaftliche) Mitte», wo auch immer sie sich befindet, ein Schutzraum der Demokratie sei. Stattdessen ist sie selbst ein sehr fragiles Gebilde, von dem eine wesentliche Bedrohung für die Demokratie ausgeht. Durch unsere Studienreihe und natürlich auch durch die Langzeituntersuchung von 2001 bis 2011 zur Gruppenbezogenen Menschenfeindlichkeit von Wilhelm Heitmeyer (deutscher Soziologe; Anm. d. R.), bekannt geworden als «Deutsche Zustände» wurde es sichtbar, wie weit die Ressentiments und autoritäre Aggressionen in der Bevölkerung verbreitet waren, wie stark eine autoritäre Dynamik in der Gesellschaft wirkt. Erst mit den Befunden von Heitmeyer und uns wurde deutlich, dass das Bedrohungspotenzial sehr viel grösser und nicht etwa an irgendwelchen politischen Rändern zu finden ist. Spätestens mit den Anschlägen während der Fluchtbewegungen nach Europa 2015 und mit den daraus resultierenden Wahlerfolgen der völkisch-nationalistischen Partei AfD kann diese Bedrohung auch jeder sehen.

Der Mitte-Begriff hatte seine Stärke, weil wir ihn mit dem Begriff des Rechtsextremismus verbanden und mit diesem scheinbaren Paradox sowohl die Gefahr aus dem Zentrum der Gesellschaft, als auch auf den ideologischen Gehalt der Mitte-Anrufung sichtbar machten. Das war nicht neu. Es gab durchaus schon die pointierte Formulierung des «Extremismus der Mitte», nämlich von Seymour Martin Lipset (US-amerikanischer Soziologe; Anm. d. R.), an die wir uns bis zu einem gewissen Grad angelehnt haben. Von Theodor Geiger, einem Sozialtheoretiker aus derWeimarer Republik, stammt auch die Rede von der «Panik im Mittelstand». Zudem geht aus den Wahlanalysen zur NSDAP eindeutig hervor, dass es eine Partei mit einem starken «Mittelstandsbauch» gewesen ist. Diese Begriffe und auch die Idee, dass da etwas innerhalb der gesellschaftlichen Mitte ist, was zu einem eigenen Extremismus führt, waren bekannt. Deswegen konnten wir auch gut daran anschliessen und haben mit der Aktualisierung aber gewissermassen eine heilige Kuh geschlachtet. Es war ein Skandal, als wir den Mitte-Begriff auf 
diese Weise verwendet haben, und das trägt sicherlich bis heute dazu bei, dass wir dafür kritisiert werden. So wird häufig bemängelt, dass wir nicht genau benennen würden, wo denn jetzt diese Mitte sei oder welche soziodemografischen Merkmale typisch wären, dass jemand der Mitte angehöre. Unsere Antwort lautet dann i.d.R., dass dies der Frage an Max Weber gleichkäme, doch bitte mal ein Passbild und die Adresse des Calvinisten rauszurücken. Weber würde daraufhin vermutlich auf seine Beschreibung einer Methode verweisen und deutlich machen, dass es nicht um einen tatsächlich empirisch vorkommenden Calvinisten, sondern um einen Idealtypus gehe, um in seinem Fall den Funktionszusammenhang von Religion und Kapitalismus auf den Punkt zu bringen. In der empirischen Welt, so schreibt Weber ausdrücklich, kann man diesen «Calvinisten» nicht antreffen. So ähnlich ist das mit unserem Mitte-Begriff, er ist eine kritische Typologie.

Es ist ja auch allen Beteiligten in der Politik klar, dass wenn sie für sich in Anspruch nehmen, eine «Partei der Mitte» zu sein, sie keinesfalls nur bestimmte Einkommensgruppen repräsentieren wollen. Innerhalb der Politik hat der MitteBegriff, was durchaus fortschrittlich ist, den Volksbegriff abgelöst. Demokratische Parteien wollen heute i.d.R. nicht mehr Volksparteien sein - das will nur die AfD sondern Parteien der Mitte. Willy Brandt hatte in den Wahlkämpfen der 1970er Jahre diese «Mitte» als erster adressiert und bis heute soll bei Wahlkämpfen ausdrücklich die «Mitte» erreicht werden. Man erkennt hier die aristotelische Mischform: nämlich die Anrufung einer gemässigten Mitte, die sich mehr durch Mass und Mässigung und weniger durch ökonomische Merkmale auszeichnet. Sie wird als Garant der herrschenden Ordnung angesprochen und soll ihre Repräsentantinnen in gemässigten Parteien haben. Damit geraten aber die Parteien in einen Konflikt. Im Grunde wissen auch die Repräsentanten der demokratischen Parteien, dass ihre Rede von der «gemäßigten Mitte» mehr Anrufung als Realität ist. Wenn sich der ehemalige SPD-Vorsitzende Sigmar Gabriel 2019 öffentlich echauffiert, dass man doch nicht von der «Mitte» sprechen solle und damit die Träger der Demokratie beschimpfen, geschieht das vor diesem Hintergrund. Die SPD weiss, dass auch und gerade ihre Anhänger undWählerinnen über Jahrzehnte schon rechtsextreme Ressentiments hatten, diese aber weder an der Wahlurne noch auf der Strasse zum Tragen kamen. Will sie diese Schichten zurückgewinnen, darf sie sie nicht damit zumuten, dass eine liberale Demokratie aus mehr besteht als exklusiver Solidarität.

Und obwohl wir unser Anliegen nun wirklich ausreichend und oft expliziert haben, werden wir immer wieder mit grosser Absicht missverstanden, wie zuletzt in der Neuen Züricher Zeitung vom 18.01.2019, als der Chemnitzer Extremismustheoretiker Ekhard Jesse unsere Studie «Flucht ins Autoritäre» anging. 
Er weiss es besser und dennoch kommt auch er nicht ohne das Lamento aus, wir würden keinen empirischen Beleg für die Existenz einer extremen Mitte liefern. Derartige Anwürfe waren aber nicht der Grund für uns, nun vom Mitte-Begriff etwas Abstand zu nehmen. Vielmehr zeigt sich, dass der «Mitte»-Begriff aus sich heraus keine weitere analytische Stärke hat, mit der die gesellschaftlichen Ursachen des Ressentiments im Sinne einer kritischen Wissenschaft verstanden werden können.

Daher haben wir auch mit der Zeit immer mehr versucht, den theoretischen Rahmen der Kritischen Theorie und psychoanalytischen Sozialpsychologie stärker zu betonen. So haben wir nun auch den Begriff der Mitte für die Studienreihe aufgegeben. Wir behalten ihn immer noch als wichtigen Begriff, doch braucht es aktuell niemanden mehr, der noch extra Warnhinweise aufstellt, damit das antidemokratische Potenzial in der Gesellschaft wahrgenommen wird. Mit Pegida, den Brandanschlägen auf Flüchtlingsunterkünfte und den Wahlerfolgen der AfD wurde es für jeden sichtbar. Damit war der Zeitpunkt gekommen, einen stärker theoretisch fundierten Begriff in den Diskurs einzubringen, sodass wir unsere Studie in «Leipziger Autoritarismus-Studie» umbenannt haben. Ob der Begriff sich durchsetzen wird, muss man schauen. Ich habe die Erfahrung gemacht, dass zwar der analytische Gehalt verstanden und auch gewürdigt wird, aber «Autoritarismus» für ungeübte Zungen doch recht sperrig ist. Bei Radiointerviews o.ä. stolpern selbst professionelle Sprecher und Journalist/innen häufig über ihn.

Laura Wolf: Über die Jahre hinweg habt Ihr in Euren Ergebnissen verschiedene Charakteristika jener Mitte hervorgehoben. Ihr sprecht 2012 bspw. von der «Mitte im Umbruch», 2014 von der «stabilisierten Mitte». Für 2016 habt Ihr jedoch den alarmierenden Titel «Die enthemmte Mitte» gewählt. Inwiefern lässt sich eine Enthemmung (in) der Gesellschaft feststellen und inwieweit setzt sich dies in Euren Ergebnissen 2018 fort?

Oliver Decker: 2014 haben wir einen sehr starken Rückgang rechtsextremer Einstellungen innerhalb der Bevölkerung dokumentiert, nachdem sie zwischen 2008 und 2012 kontinuierlich anstieg. Insbesondere im Osten, aber auch im Westen stabilisierten sie sich während der Finanz- und Wirtschaftskrise auf einem hohen Niveau und wir titelten 2010 «Die Mitte in der Krise» bzw. 2012 «im Umbruch». Mit dem rapiden Rückgang wählten wir für die Veröffentlichung 2014 die Formulierung von der «stabilisierten Mitte», die nach Jahren der ökonomischen Krisen-Rhetorik Deutschland als «Wachstumsmotor Europas» wahrnahmen. So waren unter Nützlichkeitsgesichtspunkten (Stichworte «Facharbeitermangel» und «DemografischerWandel») auch Migrant/innen willkommen - sofern sie zur nati- 
onalen Prosperität etwas beitrugen. Gingen die generalisierten Vorurteile gegen Migrant/innen zurück, so bekamen aber bestimmte Gruppen die volle Wucht der autoritären Aggression ab: Sinti und Roma sowie Muslime.

Die Untersuchung 2016 planten wir dann unter dem Eindruck der massiven Reaktionen auf die Fluchtbewegung nach Europa 2015: politisch motivierte Kriminalität, Pogrome, Brandanschläge, Körperverletzungen gegen Migranten, Erstarken der AfD u.v.m. Wir fragten uns, inwiefern wir wieder mit einer sichtbaren Zunahme rechtsextremer Einstellungen rechnen müssen; oder ob diejenigen, deren Ressentiments wir seit Jahren schon dokumentieren, die aber bisher nicht durch ein entsprechendes Verhalten auffällig wurden, jetzt dazu übergingen, zu handeln wie sie dachten? Immerhin wussten wir durch die Berichte des Bundeskriminalamts, dass diejenigen welche bspw. bei Brandanschlägen auf Asylbewerber/innenheime ermittelt werden konnten, oft bis zu diesem Zeitpunkt nicht mal wegen Fahren ohne gültigen Fahrschein auffällig geworden waren. Das waren Ersttäter jenseits jeglicher Form von Delinquenz einschliesslich politisch motivierter Kriminalität. Bis 2014/2015 fühlten sich auch die meisten rechtsextrem Eingestellten durch die grossen demokratischen Parteien repräsentiert und machten ihr Kreuz bei der SPD oder CDU. Um nicht nur die Einstellung, sondern auch die Veränderung in der Handlungsbereitschaft erheben zu können, nahmen wir zwei Fragebögen auf, die wir eine Dekade vorher in unserer Erhebung 2006 eingesetzt hatten. Mit einem Fragebogen erhoben wir die Legitimation des politischen Systems in Form von Vertrauen in ausgewählte gesellschaftliche und politische Institutionen, mit einem anderen Fragebogen versuchten wir die Befürwortung von Gewalt als Mittel der Durchsetzung von Interessen zu erfassen. Dies geschah auf zwei Dimensionen: Akzeptanz von Gewalt (Beispielitem: «Ich würde selbst nie Gewalt anwenden. Aber ich finde es gut, wenn es Leute gibt, die auf diese Weise für Ordnung sorgen») und eigener Bereitschaft zu Gewalt (Beispielitem: «Man muss leider zu Gewalt greifen, weil man nur so beachtet wird»).

Unser Ziel war es, nicht nur die Veränderung der Ressentiments in der Bevölkerung, sondern die Veränderungen in den unterschiedlichen politischen Milieus zu beschreiben. So stellten wir fest, dass zwar die demokratischen Milieus über die Dekade zwischen 2006 und 2016 angewachsen sind und in diesen demokratischen Milieus - das ist auch eine gute Nachricht - stieg die Akzeptanz des demokratischen Systems im Sinne von Vertrauen in die gesellschaftspolitischen Institutionen. Doch klafften die politischen Milieus 2016 stärker auseinander als noch 2006. Diejenigen, in denen eine ressentimentgeladene Atmosphäre aus rechtsextremen, autoritären und antidemokratischen Einstellungen herrscht, 
wurden kleiner und gleichzeitig radikaler. In diesen Milieus verlor das politische System in dem Zeitraum massiv an Legitimation. Gleichzeitig wuchs dort erstens die Befürwortung von Gewalt und zweitens auch die Akzeptanz von Gewalt als Mittel der Auseinandersetzung. In einem Milieu, dem rebellisch-autoritären, hatte sich auch die Bereitschaft entwickelt, für die eigenen politischen Ziele selbst Gewalt anzuwenden. Dieses Milieu umfasste 2016 ca. 8 bis 9 Prozent der Bevölkerung. In den anderen autoritären Milieus lebten immerhin 30 Prozent der Bevölkerung und in diesen Milieus fanden die autoritären Rebellen für ihre Gewaltbereitschaft jene Akzeptanz, die sie brauchten. Wegen dieser Radikalisierung wählten wir für unseren Bericht 2016 die Formulierung der «enthemmten Mitte». Lange Zeit gab es eine Hemmung, die dazu führte, dass die autoritäre Orientierung nicht zum Handeln führte. Heute ist es in diesen Milieus legitim, für das Ziel einer geschlossenen und autoritären Gesellschaft zu kämpfen.

Wir haben damit nicht von einem psychoanalytischen Begriff der Hemmung oder als Gegenpol einer Enthemmung gesprochen. Soweit ich das überblicke, würde im Kleinianischen Verständnis von einer Hemmung der Symbolisierungsfähigkeit gesprochen, insbesondere jener der Aggressionen beim Kind; der von uns als Enthemmung bezeichnete Vorgang ist hier ja tatsächlich überhaupt nicht der Fall. Wenn man einen psychoanalytischen Begriff heranziehen möchte, würde sich wahrscheinlich anbieten, von einer Regression zu sprechen. So ist die Enthemmung, die wir meinen, keinesfalls die einer Symbolisierung von Aggressionen, sondern ein Ausagieren der autoritären Aggression und das kennzeichnet, wenn man es genau nimmt, eher eine Form von Regression. Wir hatten kurz überlegt, ob wir die diesjährige Studienveröffentlichung statt «Flucht ins Autoritäre» mit «Regression ins Autoritäre» betiteln. Das haben wir dann aber verworfen. Wir hätten dann zwei Fremdwörter im Titel untergebracht, die für viele nicht mehr zu verstehen gewesen wären. Das autoritäre Syndrom zeichnet sich ja durch zwei sehr prominente Merkmale aus: zum einen gibt es den Wunsch nach einer Autorität, die, wie Fromm das nannte, «Prothesensicherheit» bietet, nach einer Repräsentation durch eine Autorität, nach autoritären Strukturen, die klare Ordnungen liefern, die es gestatten, scheinbare Abweichungen zu verfolgen. Und zum anderen die autoritäre Aggression, die aus dem immer ambivalenten Verhältnis zur Autorität resultiert.

Laura Wolf: In Eurer 2016er-Studie schriebt Ihr u. a. von der Liberalisierung der Gesellschaft seit den 70er Jahren, die viele Menschen aus dem Korsett normativer Rollenerwartungen und der Stigmatisierung befreit hätten. Die 68erBewegung, die sich 2018 nun zum 50. Mal jährt, wird auch häufig mit dem Begriff der «Enthemmung» in Verbindung gebracht. Inwiefern bestehen - zur begriff- 
lichen Bestimmung - Unterschiede oder Gemeinsamkeiten zwischen jener «Enthemmung» und der «Enthemmung», die Ihr adressiert?

Oliver Decker: Aus meiner Sicht sind das ganz unterschiedliche Phänomene. Man könnte dazu den psychoanalytischen Begriff anlegen, dass man sagt, es gibt eine Hemmung eines eigentlichen Bedürfnisses, das nicht zur Umsetzung kommen kann. Wenn die Hemmung jedoch wegfällt, kann es zur Bedürfnisbefriedigung kommen. Durch die gesellschaftlichen Bewegungen nach '68 und die Auseinandersetzung in den 70er Jahren, über die bleiernen 80er Jahre hinweg, könnte man zunächst von einer Enthemmung sprechen. Das wäre der Wegfall der Hemmungen individueller Bedürfnisse durch soziale Normen, die in der psychischen Struktur repräsentiert sind, also im Über-Ich oder im Ich-Ideal verankert sind. Wer die Wünsche und Bedürfnisse kontrollieren kann, entscheiden kann, was zulässig ist und was verachtet, hat ein starkes Machtinstrument in der Hand. Je liberaler eine Gesellschaft ist, desto mehr Bedürfnisse dürfen gelebt werden, desto weniger Zwangsapparaturen richten die Individuen zu, entfremden sie von ihren Wünschen und machen sie anfällig für autoritäre Dynamiken. Im Zuge der Entwicklung über die letzten 50 Jahre sind viele repressive Momente der bürgerlichen Moral im postfaschistischen Deutschland weggefallen. Doch würde ich bei dieser Entwicklung nicht von Enthemmung sprechen. Das hat sofort einen pejorativen Charakter und man liefe Gefahr, in einen denunziatorischen Diskurs einzuschwenken. Tatsächlich trifft es die «repressive Entsublimierung» eher, um in Erinnerung zu behalten, dass mit dem Wegfall des vom Vater repräsentierten Zwangssystems nicht das Reich der Freiheit begonnen hat. An dieser Stelle täuschten sich die 68er, als sie in der befreiten Sexualität bereits das Aufscheinen einer befreiten Gesellschaft verstanden. Vielleicht ist das Sexualitätsverständnis der Gegenwart, als Ort der Macht und Gewaltausübung, nicht zuletzt diesem Missverhältnis zwischen Wunsch nach Emanzipation und der weiterhin stattfindenden Reproduktion von Herrschaft geschuldet.

So gibt es auch Menschen, die mit einer solchen notwendig immer unvollständigen Emanzipation von gesellschaftlichen Zwangsstrukturen keinesfalls einverstanden sind und dies wahrscheinlich aus einer doppelten Bewegung heraus. So wünschenswert es ist, dass die Gesellschaft eine freie Entfaltung der Bedürfnisse gestattet, so wenig ist mit dem Wunsch diese Freiheit schon Realität. Und so schwer kann es für Menschen sein, dieses Spannungsverhältnis auszuhalten: wenn sie lebensgeschichtlich weder die Chance hatten, ihre Bedürfnisse zu entwickeln, noch ein stabiles Ich auszubilden, welches als vermittelnde Instanz zwischen Fremd und Eigen fungieren kann, das gestattet, die Ambiguität zwischen Wunsch und Realität 
auszuhalten, hat man von der Freiheit wenig. Die Schwierigkeit besteht zudem für den Einzelnen darin, ohne das Korsett der Konventionen eigene Bedürfnisse integrieren zu müssen. Die Schwäche des Ichs, eigene Anteile differenziert wahrzunehmen gegen die gesellschaftlichen Anforderungen in Stellung zu bringen, stellt eine beständige Herausforderung dar. Je schwächer das Ich, desto bedrohlicher und verlockender wird derWunsch. Triebtheoretisch und in einer durch den ödipalen Konflikt geprägten Gesellschaft hatte man häte man vielleicht gesagt: Er droht das Ich zu überfluten. In einer post-ödipalen Gesellschaft trifft noch etwas anders zu, nämlich die Lockung einerVerschmelzung mit dem Ich-Ideal. Die Angst genauso wie der Wunsch macht eine stabilisierende Prothese für das Ich notwendig. Sie wird nicht selten in der autoritären Dynamik gefunden, weil die autoritäre Aggression es gestattet, das Bedrohungserleben und den Wunsch abzuwehren, weil er bei «Anderen» bekämpft werden kann. Gleichzeitig wird in der autoritären Masse die Verschmelzung mit dem Ich-Ideal - zumindest phantastisch - erreicht. Der Wunsch kann dann auf dem einen oder dem anderen Weg für einen kurzen Moment zu seiner Geltung kommen: Wer nach verbotenen Düften schnüffelt, um sie auszumerzen, kann dieser Lust ungehemmt nachgehen, schrieben Horkheimer und Adorno in der Dialektik der Aufklärung. Und in der Masse steht dem phantastischen Grandiositätserleben nichts mehr im Wege.

Die Liberalisierung der letzten zwei Jahrzehnte hat paradoxerweise diese autoritäre Dynamik befeuert. Sie hatte dabei zwei Gesichter: Was wir als Neoliberalismus bezeichnen und was wir als deliberative Demokratie benennen. Während das erste über die Jahre traditionelle Schutzräume beseitigt hat, hat das zweite für eine wachsende gesellschaftliche Teilhabe gesorgt. Doppelte Staatsbürgerschaft, Kinderrechte, gewaltfreie Erziehung, Frauenrechte, die Rechte von Homosexuellen usw. das ist alles in den letzten 20, 30 Jahren in Gesetzestexte gegossen worden und wird von den meisten Menschen als Fortschritt begriffen selbst wenn sie nicht unmittelbar davon betroffen sind.

Aber Menschen sind eine Art paradoxe innere Umwelt der Gesellschaft sie sind der Gesellschaft entsprungen, aber als Entsprungene haben sie einen Eigensinn. Und dieser hat auch ein gewisses Mass an Nachträglichkeit. Somit gibt es auch Bevölkerungsteile, die den Fortschritt für sich nicht sehen können. Wer sein eigenes Leben nicht leben kann, hasst das Leben der Anderen. Und alle jene, die Fromm schon als ideale Objekte der autoritären Aggression ausmachte - Frauen, Kinder, Tiere, Fremde - haben nun auf einmal dieselben Rechte, wie jene, die sich zeitlebens den Normen unterworfen haben. Und weil auch unter den gegenwärtigen Bedingungen ja strukturelle Gewalt und Unterwerfung nicht etwa obsolet 
sind - Stichwort repressive Entsublimierung - ist das Misstrauen gegenüber den Neuerungen keine vollständige Realitätsverkennung. Daher stammt wahrscheinlich der Wunsch, auf Älteres zurückzufallen: Auf das Ideal einer Gesellschaft mit klarer Ordnung, in der die Macht lokalisierbar und eine klare Orientierung gegeben ist, in der eine personale Autorität zwar Zwang ausübt, aber ihre Milde anrufbar ist und für die Unterwerfung unter ihre Regeln Selbstwert und Stabilität bieten kann und in der autoritäre Aggressionen institutionalisiert ausgelebt werden können.

Laura Wolf: Die Veröffentlichungen Eurer Ergebnisse lösen regelmässig ein grosses Medienecho aus. Oben erwähntest Du bereits die wiederkehrende Kritik an Eurer Studie, was man bei der diesjährigen Veröffentlichung erneut beobachten konnte. 2016 seid Ihr sogar als «enthemmte Wissenschaftler» bezeichnet worden. Wieso glaubst Du, fallen die Reaktionen teils so heftig aus?

Oliver Decker: Die Reaktionen fielen eigentlich immer heftig aus, doch nahm es tendenziell ab. Zumindest im bürgerlich-konservativen Lager geht es eher zurück. Massive Reaktionen, auch von regierungsnahen Institutionen initiiert, erlebten wir das erste Mal 2006.

Laura Wolf: Im Jahr der Fussballweltmeisterschaft in Deutschland...

Oliver Decker: (lacht) Das kann sein. Mit Fussball kenne ich mich nicht so aus. Doch hingen die Reaktionen meines Erachtens damit gar nicht so sehr zusammen. Klar, bei dieser Weltmeisterschaft wurden wieder vermehrt deutsche Fahnen geschwenkt und man fuhr ungeniert mit Fahnenüberziehern auf seinen Autoaussenspiegeln herum, kurz: man konnte die Rückkehr eines offenkundigen Nationalismus in Deutschland beobachten. Aber die Reaktionen auf unsere damalige Veröffentlichung kamen noch aus ganz anderer Quelle. Sie ging darauf zurück, dass wir feststellten, dass wir bei den rechtsextremen Einstellungen zwar einen sehr klaren Ost-West-Unterschied mit hohen Werten in Ostdeutschland haben, aber im Freistaat Bayern vergleichbar und konstant bis heute hoheWerte ähnlich wie bspw. in Mecklenburg-Vorpommern vorfinden. Bayern ist ein Bundesland, in dem rechtsextreme Einstellungen sehr verbreitet sind, und das war ein Skandal. Das fanden nicht nur wir, sondern auch die bayrische Landesregierung. Günther Beckstein meldete sich öffentlich damit zu Wort, dass unsere Studie wertlos sei. Aber dabei sollte es nicht bleiben. Über die Landeszentrale für politische Bildung gab die CSULandesregierung ein Gegengutachten bei einem Politikwissenschaftler in Auftrag. Der Berliner Politologe Klaus Schroeder war bereits häufiger für die Landeszentrale tätig gewesen und erstellte nun im Auftrag und mit einem Honorarvertrag ausgestattet eine Stellungnahme. Solcherart Vorgehen und zumal das äusserst schwache Ergebnis eines in der empirischen Sozialforschung selbst völlig unerfahrenen 
Wissenschaftlers war selbst in der bayrischen Landesvertretung nicht unumstritten. Der damalige Leiter der Landeszentrale bot uns im Gegenzug an, zum gleichen Honorar ebenfalls eine Stellungnahme zu schreiben. Zum Glück brachten sich aber schon ohne unser Zutun unterschiedliche Wissenschaftlerinnen und Wissenschaftler ein, die klar und deutlich machten, dass Klaus Schroeder weder vom Forschungsfeld des Rechtsextremismus noch von empirischer Sozialforschung Kenntnis, geschweige denn eine Ahnung hat. Tatsächlich kommen Gegengutachten, die wir weder selbst in Auftrag gegeben haben noch sonst wie beeinflusst haben, zu dem Schluss, dass die Lesart Schroeders bösartig sei. Trotzdem liefert er bis heute die Standard-Situation der Kritik an unserer Studie.

Zumeist wird darauf hingewiesen, dass man nicht bei allen Aussagen unseres Fragebogens rechtsextremer Einstellungen sofort an einen rechtsextremen Inhalt denken könne. Dabei sind die Lesarten der «Kritiker» jedoch sehr unterschiedlich. Mal wird einer Aussage unseres Fragebogens sofort zugestanden, dass sie eine rechtsextreme Einstellung abbildet, anderen dagegen wird genau dieser Gehalt abgesprochen. Es folgt dann oft eine Textexegese. Zum Beispiel könne, so eine vorgebrachte Kritik, die Aussage «Es gibt wertvolles und unwertes Leben» genauso gut auf eine Mücke bezogen werden und offenbare daher keinesfalls rechtsextremes Gedankengut. Das ist offensichtlich an den Haaren herbeigezogen, weil wir weder denjenigen als rechtsextrem bezeichnen, der einer einzelnen Aussage zustimmt noch es zutrifft, dass diese Aussagen falsch verstanden werden. Der Fragebogen misst ein Konstrukt und weist eine hohe teststatistische Güte auf. Jemand, der die Aussage «Es gibt wertvolles und unwertes Leben» zustimmend beantwortet, beantwortet in der Tendenz auch antisemitische Aussagen zustimmend und diejenigen mit ausländerfeindlichem Inhalt. Der Fragebogen hat eine hohe interne Konsistenz und die Kennwerte sind auch publiziert. Diese wissenschaftliche Güte ändert aber nichts an solchen Einlassungen und ich vermute, dass auch die einzige Wortmeldung, die dieses Jahr in jener Richtung der Kritik wieder aus der Frankfurter Allgemeinen Zeitung zu verzeichnen war, nicht nur in Unkenntnis dieser Forschungsmethoden vorgebracht wurde, sondern auch weil ein konservativer nationaler Grundkonsens mitberührt wird. So hat beispielsweise die ehemalige Familienministerin Kristina Schröder (CDU, 2009-2013 Bundesministerin für Familie, Senioren, Frauen und Jugend; Anm. d. R.) unseren Fragebogen im Bundestag vorgelesen und ihre applaudierenden CDU-Kollegen gefragt, was denn daran rechtsextrem sei.

Laura Wolf: (lacht) Frau Schröder hat damit die heilige Kuh im Bundestag inszeniert. 
Oliver Decker: Ja, es werden mit unseren Fragen eben auch Inhalte aufgerufen, mit denen sich auch national-konservative Kreise identifizieren können. Das ist ein Teil des Skandals und des Ärgernisses.

Laura Wolf: Methodologisch zieht Ihr bei Eurer Forschung ähnlich wie ein/e Psychoanalytiker/in individuelle Äusserungen heran, doch gelangt Ihr zu gesamtgesellschaftlichen Aussagen. Was ist bei der sozialwissenschaftlichen Nutzung psychoanalytischer Theorie zu beachten?

Oliver Decker: Das ist schwierig in Kürze zu beantworten. Ich würde vielleicht bei Wilhelm Dilthey ansetzen, auf den die Unterscheidung zweier Wissenschaften zurückgeht: die erklärende und die verstehende Psychologie. Diese Unterscheidung hat im Grunde genommen bis heute Gültigkeit, auch wenn wir gewohnt sind, heute eher zwischen qualitativer und quantitativer Forschung zu unterscheiden. Angelehnt an die Naturwissenschaften sucht die erklärende Psychologie Zusammenhänge theoretisch zu erklären und die Erklärung anschliessend empirisch zu überprüfen. Die gebildeten Theoriemodelle werden in Form von Hypothesen überprüft bzw. wenn wir dem Kritischen Rationalismus Karl Poppers folgen, falsifiziert. Die verstehende Psychologie versucht Bedeutungszusammenhänge von Handeln und Erleben zu rekonstruieren. Die Psychoanalyse teilt dieses Erkenntnisinteresse. Und durch die Psychoanalyse wurde mit dem Unbewussten eine weitere Bedeutungsdimension in den Verstehensprozess einbezogen. Mit der Kritischen Theorie, wurde noch ein drittes Erkenntnisziel formuliert, das über dieses Moment des Erklärens oder Verstehens hinausgeht. Deshalb würde ich auf jeden Fall zwischen einem nomothetisch-erklärenden, hermeneutisch-verstehenden und kritisch-emanzipativen Erkenntnisziel unterscheiden. Für letztere formulierte Max Horkheimer 1937 das Erkenntnisziel: «vom Interesse an vernünftigen Zuständen durchherrschte kritische Theorie der bestehenden Gesellschaft». Das ist auch unser Anspruch, ob wir ihn einlösen können, ist eine andere Frage, aber wir gehen davon aus, dass eine Kritische Theorie der Gesellschaft auch auf das Instrumentarium der erklärenden Forschung zurückgreifen kann und sollte.

Von psychoanalytischer Seite als auch von Seiten qualitativer Forschung her wird häufig an der quantitativen Forschung kritisiert, dass sie dem Gegenstand nicht gerecht würde, weil die Bedeutungsdimension, die das Individuum zum Handeln oder Motiven zugrunde legt, mit einem Fragebogen nicht erfasst werden könne. Das ist nicht grundsätzlich falsch, verkennt allerdings, dass wir auch mit einem Fragebogen durchaus Qualitäten erheben, zum Beispiel Stimmung, Empfindung, Wahrnehmung; natürlich in ihrer Ausprägung, also Häufigkeit und Stärke des Merkmals, aber es bleiben Qualitäten. Wir bilden auf diese Weise 
ebenso einen subjektiven Blick auf die Welt ab, denn auch die Objektivität eines Fragebogens ist nur seine Verfahrensobjektivität. Wir stellen sicher, dass jeder dieselbe Anleitung bekommt und dieselben Fragen beantwortet. Was wir aber dann von Individuen berichtet bekommen, sind subjektive Sichtweisen auf dieWelt. Also das Item «Finden Sie die Politiker und andere Führungspersönlichkeiten sind nur Marionetten der dahinterstehenden Mächte?» bildet einen subjektiven Blick auf die Welt ab und wir erheben dieses in seiner Stärke bzw. der Ausprägung.

Dass uns das gelingt, würde nicht überall und zu allen Zeiten funktionieren. Fragebögen zu beantworten ist eine Kulturtechnik, die erlernt werden muss. Und die auch erlernt wird. Zum Beispiel haben wir mit Geflüchteten Befragungen gemacht und gesehen, dass es u.a. Schwierigkeiten mit dem Format eines Fragebogens gibt. In Europa hat sich eine instrumentelle Vernunft durchgesetzt, die es möglich macht, sowohl den Musikgeschmack als auch die eigenen Empfindungen auf einer Skala von 1 bis 6 abzubilden. Aber genau dadurch, dass Individuen den instrumentellen Blick auf sich selbst anwenden, produzieren wir nicht Artefakte, sondern erfassen diese Subjektivität. Daher arbeiten wir sowohl quantitativ als auch sinnrekonstruktiv. Wir können angeben, wie weit Ressentiments in Deutschland verbreitet sind und was ihre Ursachen sind. Man sieht dann, es gibt so und so viel Einstellungsveränderungen, die sich erfassen lassen, wie sind die Zusammenhänge. Z.B. wirkt sich die erfahrene Gewalt in der Erziehung aus, die verweigerte Anerkennung als Bürger, die nationale ökonomische Grösse?

Wenn wir Gruppendiskussionen durchführen, dann greifen wir auf das szenische Verstehen von Alfred Lorenzer zurück und können den allgemeinen Stigmatisierungsdruck in der Gesellschaft in ihrer kleinsten Einheit rekonstruieren. Im Grunde genommen lehnen wir uns bei diesen Versuchen an Weber an, der seine Methode auf den Begriff «kompilieren» gebracht hat oder an Walter Benjamins «Montagetechnik». Wir führen die Ergebnisse aus der empirischen Forschung zusammen und beschreiben so zum Beispiel die Dynamik eines «sekundären Autoritarismus» oder sprechen vom deutschen Wirtschaftswunder als «narzisstischer Plombe». Dann ist das eine Montage, wir kompilieren die soziale Wirklichkeit aus der Fülle des Materials. Dieses Vorgehen führte uns auch zum «Mitte»-Begriff, wie wir ihn verwendet haben.

Das sind Gerinnungsformen und vielleicht kann man sagen, dass dieser Erkenntnisweg den Freudschen Versuchen nicht unähnlich ist. In der Dyade der psychoanalytischen Therapie wird auch eine Montage oder Konstruktionsleistung vorgenommen. Dem heutigen Verständnis nach wird diese Konstruktionsleistung adhoc im Prozess vollzogen und deren Gültigkeit zur Überprüfung besteht im 
Grunde genommen in der Enthemmung, wenn wir den Begriff nochmals aufnehmen; nämlich, dass die Hemmung, die einen Menschen daran hindert, liebesund arbeitsfähig zu sein, aufgehoben wird. Genau an dieser Stelle muss sich die Konstruktion der Analyse bewähren, und so ähnlich ist es bei uns auch. Unsere Sinn- und Bedeutungsrekonstruktion muss sich als Kritik in einer Form von Handlungserweiterung bzw. Verständniserweiterung bewähren. Dann ist sie hoffentlich tragfähig, zumindest für den Moment. Denn Wahrheit hat einen Zeitkern.

Laura Wolf: $\mathrm{Zu}$ Beginn beschriebst Du die Anrufung der Mitte auch als einen Ort der Mässigung und bringst nun die Ökonomie als narzisstische Plombe der Bundesrepublik ein. Ich würde davon ausgehend gern in einem etwas grösseren Bogen zu den autoritären Dynamiken zu sprechen kommen, die Ihr bei Euren Erklärungen zunehmend ins Visier nehmt: Mit dem Titel «Flucht ins Autoritäre» skizziert Eure Studienveröffentlichung 2018 dies bereits und in deren ersten Kapitel triffst Du u. a. die Unterscheidung zwischen autoritärem Syndrom auf individueller Ebene und autoritärer Dynamik auf gesellschaftlicher Ebene. Dabei ziehst Du eine enge Linie zwischen Autoritarismus und Anerkennung in der Tauschgesellschaft. Die auf Wachstum zielende Ökonomie gefährde aber auf Dauer die Lebensgrundlage der Menschheit. Wie kommt es also dazu, dass sich so die häufige These - vor allem die sogenannten «Globalisierungsverlierer» den extrem rechten Bewegungen zuwenden würden? Könntest Du diesen Komplex noch mehr ausführen?

Oliver Decker: Zunächst zeigt unsere Analyse ja nur, dass Deutschlands Ökonomie in ihrer Funktion als narzisstische Plombe nicht immer gleichermassen stark ausgeprägt ist und diese erfüllen kann. Jedoch würde ich bekräftigen, dass sie eine Art Ausdruck von immer noch andauerndem Phantomschmerz wegen der verlorenen Herrenreiterideologie ist und ein Verlustschmerz aus der Schuld und der Scham über den verlorenen Angriffs- und Vernichtungskrieg ist. Das ist bis heute der Erbteil Nazi-Deutschlands: Scham- und Schuldgefühle wurden genauso verdrängt, wie der Verlust des idealisierten Objekts verleugnet. Die aus der Verleugnung resultierende Lücke musste geschlossen werden. Und das vollzog sich - man ahnt es schon, wenn der Begriff Verleugnung fällt - in dem ein anderes Objekt an die Stelle gesetzt worden ist. Ich habe dieses Begriffspaar der narzisstischen Plombe von Fritz Morgenthaler entlehnt, der bereits darauf hinwies, dass der Fetischismus vielmehr eine soziokulturelle Plombe ist als ein Einzelschicksal. Dieses konnte und kann als Status, Autorität sowohl Sicherheit als auch Selbstwert vermitteln und in die Gesellschaft integrieren. Zudem konnte damit an die Entwicklung vor dem Ende des Zweiten Weltkrieges angeknüpft wer- 
den, da das «Deutsche Wirtschaftswunder» zum ersten Mal zur Beschreibung des durch die deutsche Kriegswirtschaft befeuerten Wachstums verwendet wurde. Wie immer bei der Verleugnung geht diese Objektverschiebung mit einer teilweisen Anerkennung der Realität einher - der französische Psychoanalytiker Octave Mannoni verglich diesen Abwehrmodus in den 60ern mit dem paulinischen Glaubensbekenntnis credo qoud absurdum, oder eben auf Französisch: «Je sais bien mais quand même...». Aber bereits Morgenthaler hat darauf hingewiesen, wie unvollständig diese fetischistische Verleugnung funktioniert: die Prothese ist nicht bloss die zweitbeste Fahrt, das Bewusstsein darüber bleibt auch noch immer haften. Die narzisstische Plombe ist eben eine Plombe, die wie jede Prothese den Mangel ständig erinnert. Auf diese Doppellogik der Prothese bin ich bereits früher in meiner Dissertation «Der Prothesengott» eingegangen. Die Prothese als Hinzufügung ruft die Phantasie wach, dass der Zustand vor der Prothetisierung der eigentlich bessere war.

Der Hamburger Kollege Wolfgang Menz (deutscher Soziologie; Anm. d. R.) hat diese Idee unserer narzisstischen Plombe und des sekundären Autoritarismus aufgegriffen und vom halbierten Autoritarismus gesprochen, da die vom Einzelnen erhoffte Schutzfunktion von der Autorität des Marktes nie vollständig geleistet werden kann. Das kann sie ebenso wenig von einer tatsächlichen Führerfigur. Doch immer dann, wenn deutlich sichtbar wird, dass das Versprechen der Ökonomie Macht, Teilhabe, Grössenerleben, Aussicht auf einen versöhnten Zustand usw. nicht einzulösen ist, sondern im Gegenteil eine grosse Unsicherheit produziert, bekommt die ursprüngliche Idee einer personellen Autorität in Form eines Führers die grössere Attraktivität. Ich glaube, dass wir damit zum Teil rechnen müssen, dass dieser sekundäre Autoritarismus als Integrationskraft nachlässt. Interessant ist es, gegenwärtig zu beobachten, wie stark gleichzeitig der Wunsch nach der narzisstischen Plombe und einer Restitution vergangener Grösse ist. Im Grunde genommen verspricht die AfD zum einen mit ihrem gleichermassen ordoliberalen Programm und zum anderen mit ihrer völkischen Propaganda beides: sowohl den Gewinn aus dem Gefühl, Teil einer starken Wirtschaftsnation zu sein, als auch die Rückkehr zu alten autoritären Strukturen des primären Autoritarismus. Wie gute faschistische Agitatoren ahnen sie schlafwandlerisch, was ihr Publikum wünscht. Sie versprechen beides, selbst wenn es sich widerspricht - mais quand même-und das macht sie für bestimmte Bevölkerungsgruppen sehr attraktiv. Man hat sowohl das Gefühl, «wir» sind eine moderne Wirtschaftsnation, die solitär in derWelt steht, als auch das Gefühl, dass es autoritäre Strukturen gibt, an die man sich anlehnen 
kann. Das Nächste wäre dann eine Führerfigur, die das dann personell repräsentiert und durch Identifikation dann zum idealen Selbst-Objekt werden kann.

Laura Wolf: Kurz dazwischengefragt: In Deutschland scheinen extrem rechte Bewegungen gegenwärtig (noch) ohne vermeintlich starke Führerfigur zu funktionieren im Gegensatz zu anderen europäischen Ländern. Wie erklärst Du Dir das?

Oliver Decker: Es gibt verschiedene Nationen in Europa und in den westlichen Industrieländern, in denen eine starke Führerperson nicht zu sehen ist.

Laura Wolf: Aber Personen wie Marine Le Pen in Frankreich, Geert Wilders in den Niederlanden oder Sebastian Kurz bzw. Heinz-Christian Strache in Österreich haben unglaublich an Popularität, im letzteren Fall auch die Wahl gewonnen.

Oliver Decker: Ich würde nur mit Einschränkung sagen, dass Kurz und Strache Führerfiguren sind. Aber Du hast Recht, auch in der Vergangenheit waren faschistische Führer für den aufmerksamen Beobachter genauso Witzfiguren, wie es heute Trump eine ist. Zunächst einmal wäre wahrscheinlich die Diskussion spannend, was an den personalisierten Wahlkämpfen bereits an einem Mechanismus ansetzt, den die faschistische Agitation perfektioniert. In dieser Form, wie zur Zeit Wahlkämpfe inszeniert werden, bauen sie immer auch auf Personalisierung auf. Ganz selten wie bspw. bei vielen Wahlkämpfen der Grünen ist das nicht der Fall. Es ist kein Kennzeichen einer faschistischen Bewegung, dass es einen personalisierten Wahlkampf gibt. Dann hätte man ziemlich viele faschistische Bewegungen. Doch kann das Extrem der faschistischen Bewegung nicht verstanden werden, ohne den Regelfall zu verstehen. Es gibt offensichtliche Gemeinsamkeiten, die bestehen nicht zuletzt in den Bedürfnissen der Bevölkerung, und dann gibt es Übergänge.

Zum Beispiel kann man Viktor Orbán, Ministerpräsident Ungarns, Leo Löwenthal folgend, durchaus als einen faschistischen Agitator bezeichnen, Donald Trump auch. Sie sind nicht die Zauberkünstler, für die sie sich selbst halten, sondern der Spuk, den ihre Zuhörer, alles kleine Zauberlehrlinge, heraufbeschwören und dann doch nicht im Griff haben. Die französische Psychoanalytikerin Janine Chassguet-Smirgel hatte darauf hingewiesen, welche Rolle der Führer spielt: Der Führer ist der Mittelsmann zwischen Masse und Illusion, schreibt sie, und meint die Illusion einer Verschmelzung mit dem Idealen Ich. Löwenthal beschreibt schon 1949 auf faszinierende Weise, wie der faschistische Agitator sich an die Bedürfnisse seiner Anhänger herantastet und wer das liest, hat Trump unmittelbar vor Augen, genauso wie den deutschen AfD-Politiker Höcke. Noch ist dies in Grossbritannien oder in Spanien nicht der Fall, wobei stattdessen dort offensichtlich das Bedürfnis über eine sekundäre Autorität befriedigt wird: die «Nation». 
Die Situation in Portugal finde ich übrigens vernachlässigt und man müsste mal untersuchen, was dort der Grund ist, dass es diese Bedürfnisse scheinbar nicht gibt. In Deutschland gibt es zwar Bewerber für den Job des Agitators, aber entweder läuft das Casting noch oder der Bedarf nach einer solchen personellen Führerfigur ist noch nicht so deutlich, weil einerseits weite Teile der Bevölkerung mit der liberalen Demokratie identifiziert sind und anderseits die Identifikation mit der sekundären Autorität noch funktioniert. Ob das auf Dauer so bleibt? Wir wissen aus der jüngeren Vergangenheit eines postfaschistischen Deutschlands und der Gegenwart, wie schnell wirtschaftliche Krisen dazu führen, dass der Wunsch nach einer autoritären Lösung aufkommen kann. Denken wir mal an die erste Wirtschaftskrise nach dem Krieg 1967 zurück, die sofort zum Einzug der NPD in die westdeutschen Parlamente führte. Die letzte Finanzmarktkrise 2008 und die anschliessende Wirtschaftskrise führten, wie schon gesagt, zum rapiden Anstieg der rechtsextremen Einstellungen in der Bevölkerung. Ich würde vermuten, dass wenn die nächste Krise kommt, dann werden wir auch erneut mehr Zustimmung zu rechtsextremen Aussagen verbuchen müssen. Und sie wird kommen, denn die Krise gehört zum Kapitalismus wie der Herbst zu den Jahreszeiten.

LauraWolf: Jetzt sind wir bereits bei den Prognosen angelangt. Wenn gerade die Krisen wie der Herbst kommen, wieso schaffen es dann linke Bewegungen wie die zum Beispiel von Sahra Wagenknecht (Fraktionsvorsitzende DIE LINKE im deutschen Bundestag) gegründete Sammlungsbewegung «Aufstehen» nicht, derartige Massen zu mobilisieren? Wäre das nicht auch ein mögliches prognostisches Szenario?

Oliver Decker: Ich würde sagen nein. Die Hoffnung ist gross und ich verstehe auch den Wunsch. Schon Stuart Hall (britischer Soziologie; Anm. d. Red.) hatte bei seiner Analyse des Thatcherismus und der Economics von einem rechtsautoritären Populismus als neoliberales Krisenmanagement gesprochen, dem ein demokratischer Populismus entgegenzustellen sei. Ähnlich argumentiert auch Chantal Mouffe (belgische Politikwissenschaftlerin; Anm. d. R.). In diesen Analysen ist die rechte Propaganda vergleichbar mit einem Priesterbetrug, auf den die Zuhörer hereinfallen. Genau das ist aber faschistische Propaganda nicht oder nicht zuerst. Das kann man an Sahra Wagenknechts Versuch sehen. Auch ihr schien es nicht anders möglich als unter Nutzung des Ressentiments, also nicht ohne, dass sie sich auch xenophobe Parolen zum Teil aneignet, um für eine progressive Politik zu werben. Ein faschistischer Agitator reagiert auf die Bedürfnisse des Publikums. Will man als progressive Politikerin mit ihm konkurrieren, läuft man schnell Gefahr, mit den eigenen aufgeklärten Zielen in Widerspruch zu geraten. 
Laura Wolf: Das klingt sehr pessimistisch.

Oliver Decker: Nicht ganz, wir sollten vielmehr kritisieren, wie diese Bedürfnisse entstehen. Das ist jetzt nicht nur triebpsychologisch gemeint, also nicht nur als Analyse der Bearbeitung menschlicher Bedürfnisse. Es gibt das Bedürfnis nach Kontrolle über relevante Lebensbereiche und damit verbunden ein Bedürfnis nach Handlungsfähigkeit. Es spielt noch mehr mit hinein. Aber ich glaube, wenn wir intervenieren wollen, dann müssen wir uns überlegen, wie Handlungsfähigkeit in einem aufgeklärten Sinne und nicht in einem repressiven oder regressiven Sinne aussehen kann. Alice Pechriggl (Philosophin und Gruppenanalytikerin; Anm. d. R.) aus Klagenfurt unterscheidet zum Beispiel zwischen Handeln und Agieren und dies sowohl gruppenanalytisch als auch kritisch-wissenschaftlich betrachtet. Kein Handeln ist ohne Agieren, kein Agieren ohne das Potenzial zum Handeln, manchmal birgt gerade das Agieren progressives Potenzial, weil es die instrumentelle Rationalität unterläuft. Aber unter welchen Bedingungen Gruppen in der Lage sind, fortschrittliche politische Ziele zu verfolgen, gilt es immer wieder zu verstehen. Wie kommen wir zu einer Handlungsfähigkeit, die nicht ein Ausagieren von Verdrängtem und gesellschaftlich Unbewusstem darstellt? Mein Verdacht ist, dass Wagenknecht hin und wieder versuchte die Abkürzung zu nehmen. Sie greift dafür auch anti-solidarische Motive auf, doch kommt man damit in einen performativen Selbstwiderspruch. Das ist das Problem.

Laura Wolf: Paul Parin schrieb 1978 in der Psyche eine Glosse unter dem Titel «Warum die Psychoanalytiker so ungern zu brennenden Zeitproblemen Stellung nehmen». 2007 unterziehst Du mit weiteren Autoren diese Diagnose einer Wiederlese, ebenfalls in der Psyche veröffentlicht. Wäre es daher eine Interventionsmöglichkeit, wenn sich Psychoanalytiker/innen mehr zu den brennenden Zeitproblemen äussern, um auch diese Frage nach den Bedürfnissen zu adressieren?

Oliver Decker: Es tut der Demokratie immer gut, wenn sich viele Menschen einmischen. Die Demokratie könnte sicherstellen, was Adorno in der «Minima Moralia» als fast nicht zu leistende Aufgabe formulierte: sich weder von der Übermacht der Anderen noch von der eigenen Ohnmacht dumm machen zu lassen.

Psychoanalytisch informierte Eingriffe in die Öffentlichkeit wären meines Erachtens sinnvoll. Doch habe ich derzeit den Eindruck, dass wenn Psychoanalytiker/ innen eingreifen, sie dies leider nicht mit den Erkenntnismöglichkeiten tun, die die Psychoanalyse bietet. Stattdessen äussern sie sich als Sozialwissenschaftler/ innen und rekurrieren auf wissenschaftliche Literatur von bspw. Hartmut Rosa 
(deutscher Soziologe und Politikwissenschaftler; Anm. d. R.) oder Anthony Giddens (britischer Soziologe; Anm. d. R.). Dagegen ist nichts einzuwenden, doch ist es schade. Gerade Giddens konnte für seine Theoriebildung sehr gut auf psychoanalytische Erkenntnisse zurückgreifen. Es wäre jetzt vielleicht auch wieder an der Zeit, psychoanalytische Erkenntnisse für eine psychoanalytisch informierte Soziologie nutzbar zu machen und diese psychoanalytische Erfahrung in die kritische Theorie zurückzutragen. Es ist kein Zufall, dass Christoph Türcke (deutscher Philosoph; Anm. d. R.) und ich alle zwei Jahre die Tagung «Kritische Theorie Psychoanalytische Praxis» veranstalten. Damit wollen wir nicht die Psychoanalyse auf eine Praxis reduzieren, sondern ihre Erfahrungsdimension betonen und durch diese ihren Beitrag für eine Theoriebildung zur Gesellschaft herausstellen. Ich glaube, da gibt es eine grosse Lücke, die keine Einzelperson lösen kann. Es hängt auch mit einer generellen Entpolitisierung bspw. der Weiterbildungskandidatinnen und -kandidaten zusammen. Zudem geht vielleicht mit Ausnahme des PSZ die Integration in die Institutionen und die Professionalisierung zum/r Psychoanalytiker/in i.d.R. mit einer Über-Ich-Introjektion vonstatten, wodurch die Kritikfähigkeit nicht unbedingt gesteigert wird. Das ist ein grosses Problem. Auch ist von den Kandidatinnen und Kandidaten häufig kein anderer Wunsch zu verzeichnen, als die Approbation zu bekommen. Dabei könnte gerade die Psychoanalyse mit der psychoanalytischen Erfahrung enorm zum Verständnis und zur Aufklärung über die Widersprüche der Gesellschaft beitragen.

Laura Wolf: Vielen Dank für das Gespräch!

\section{Erwähnte Literatur:}

Adorno, T. W. (1980 [1954]). Minima Moralia. In R. Tiedemann (Hg.), Theodor W. Adorno-Gesammelte Schriften Bd. 4. Frankfurt a. M.: Suhrkamp.

Chasseguet-Smirgel, J. (1975). Das Ichideal. Psychoanalytischer Essay über die «Krankheit der Idealität». Frankfurt/M.: Suhrkamp (1987).

Decker, O. (2004). Der Prothesengott. Subjektivität und Transplantationsmedizin. Gießen: Psychosozial-Verlag.

Decker, O., Rothe, K., \& Brosig, B. (2007). «Wie nehmen die Analytiker zu brennenden Zeitfragen Stellung?» Paul Parins Aufsatz von 1978 wiedergelesen. Psyche, 61 (12), 1270-1280.

Geiger, T. (1930). Panik im Mittelstand. Die Arbeit.Zeitschrift für Gewerkschaftspolitik und Wirtschaftskunde, 10, 637-654.

Heitmeyer, W. (Hg.). (2002). Deutsche Zustände. Berlin: Suhrkamp. 
Horkheimer, M. (1988 [1937]). Traditionelle und Kritische Theorie. In G. Schmid Noerr (Hg.), Max Horkheimer - Gesammelte Schriften Bd. 4. Frankfurt a. M.: Fischer.

Horkheimer, M. \& Adorno, T.W. (1987 [1947]). Dialektik der Aufklärung. In G. Schmid Noerr (Hg.), Max Horkheimer - Gesammelte Schriften Bd. 5. Frankfurt a. M.: Fischer.

Löwenthal, L. (1990 [1949]). Falsche Propheten. In H. Dubiel (Hg.), Leo LöwenthalSchriften 3. Zur politischen Psychologie des Autoritarismus (S. 11-160). Frankfurt a. M.: Fischer.

Lipset, S. M. (1967 [1959]). Der «Faschismus`, die Linke, die Rechte und die Mitte. In E. Nolte (Hg.), Theorien über den Faschismus (S. 449-491). Köln: Athenäum (1967).

Morgenthaler, F. (1974). Die Stellung der Perversionen in Metapsychologie und Technik. Psyche, 28 (12), 1077-1088.

Parin, P. (1978). Warum die Psychoanalytiker so ungern zu brennenden Zeitproblemen Stellung nehmen: Eine ethnologische Betrachtung. Psyche, 32 (5-6), 385-399.

\section{Die erwähnten Studien:}

Decker, O., Kiess, J. \& Brähler, E. (Hg.). (2012). Die Mitte im Umbruch. Rechtsextreme Einstellungen in Deutschland 2012. Bonn: Dietz-Verlag.

Decker, O., Kiess, J. \& Brähler, E. (Hg.). (2014). Die stabilisierte Mitte. Rechtsextreme Einstellungen in Deutschland 2014. Leipzig: Universität Leipzig.

Decker, O., Kiess, J. \& Brähler, E. (Hg.). (2016). Die enthemmte Mitte. Autoritäre und rechtsextreme Einstellungen in Deutschland. Gießen: Psychosozial-Verlag.

Decker, O., \& Brähler, E. (Hg.). (2018). Flucht ins Autoritäre. Rechtsextreme Dynamiken in der Mitte der Gesellschaft. Gießen: Psychosozial-Verlag. Online verfügbar unter: http://home.uni-leipzig.de/decker/Flucht\%20ins\%20Autoritaere.pdf 